\title{
The price of prosociality in pandemic times
}

\author{
Hernando Santamaría-García,2,3, Miguel Burgaleta4 ${ }^{4}$ Agustina Legaz ${ }^{5,6}$, Daniel Flichtentrei ${ }^{7}$, \\ Mateo Córdoba-Delgado (1) ${ }^{8}$, Juliana Molina-Paredes ${ }^{8}$, Juliana Linares-Puerta ${ }^{8}$, Juan Montealegre-Gómez ${ }^{8}$, \\ Sandra Castelblanco (10 2, Michael Schulte ${ }^{6,9}$, Juan David Páramo ${ }^{2,3}$, Izara Mondragon ${ }^{8}$, \\ Juan David Leongómez (10 ${ }^{10}$, Paula Salamone (i] 5,11, Juan González-Pacheco ${ }^{8}$, Sandra Báez ${ }^{12}$, \\ Harris Eyre ${ }^{13,14,15,16}$ \& Agustín Ibanez (i) 5,6,9,17,18,19凶
}

The SARS-CoV-2 pandemic has imposed widespread negative impacts (economically, psychologically, neurologically, and societally), and has changed daily behaviors on a global scale. Such impacts are more significant and pervasive in countries with higher levels of inequality and reduced Government capacity and responsiveness, such as those in the Global South (e.g., Colombia). Differences in social and moral cognitive skills may significantly impact individual attitudes and responses to the pandemic. Here, we aimed to assess the extent to which factors associated with prosociality (including empathy, theory of mind (ToM), and moral judgments) predict the perception of SARS-CoV-2 impacts and responses. Participants $(N=413)$ from Colombia answered factors associated with prosociality measures and judgments about SARS-CoV-2 risk, impact, and acceptance of quarantine guidelines. Results revealed that affective empathy (personal distress and empathic concern) and moral tendencies (deontological trends) predicted greater acceptance of quarantine but in turn yielded an increased perception of risks and individual impacts of SARS-CoV-2. Moreover, age (older) and gender (female) also increased the risk perception and impact estimation. These results underscore the role of prosocial-related predispositions informing individual responses to the pandemic and provide an opportunity to exploit this knowledge to inform successful interventions favoring behavioral change.

\footnotetext{
${ }^{1}$ Program of Neuroscience, Psychiatry Department, Pontificia Universidad Javeriana, Bogotá, Colombia. ${ }^{2}$ Intellectus Memory and Cognition Center, Hospital Universitario San Ignacio, Bogotá, Colombia. ${ }^{3}$ Global Brain Health Institute (GBHI), University of California San Francisco (UCSF), San Francisco, CA, US. ${ }^{4}$ University of Barcelona, Barcelona, Spain. ${ }^{5}$ National Scientific and Technical Research Council (CONICET), Buenos Aires, Argentina. ${ }^{6}$ Cognitive Neuroscience Center (CNC), Universidad de San Andrés, Buenos Aires, Argentina. ${ }^{7}$ Intramed, Buenos Aires, Argentina. ${ }^{8}$ Pontificia Universidad Javeriana, MENTE research group, Bogotá, Colombia. ${ }^{9}$ Latin American Brain Health Institute (BrainLat), Universidad Adolfo Ibáñez, Santiago, Chile. ${ }^{10}$ Universidad El Bosque, Bogotá, Colombia. ${ }^{11}$ Center for Social and Affective Neuroscience, Linköping University, 58185 Linköping, Sweden. ${ }^{12}$ Universidad de los Andes, Bogotá, Colombia. ${ }^{13}$ Institute for Mental Health and Physical Health and Clinical Translation, Deakin University, Geelong, VIC, Australia. ${ }^{14}$ Department of Psychiatry, Baylor College of Medicine, Houston, TX, USA. ${ }^{15}$ Neuroscience-inspired Policy Initiative, Organisation for Economic Co-operation and Development and PRODEO Institute, Paris, France. ${ }^{16}$ Department of Psychiatry, University of Texas Health Sciences Center at Houston, Texas, USA. 17 Universidad de San Andrés, Buenos Aires, Argentina. ${ }^{18}$ Global Brain Health Institute (GBHI), University of California San Francisco (UCSF), San Francisco, USA. ${ }^{19}$ Trinity College Dublin (TCD), Dublin, Ireland. ${ }^{凶}$ email: agustin.ibanez@gbhi.org
} 


\section{Introduction}

he SARS-CoV-2 pandemic has severely impacted health and everyday life. Physical distancing and governmentsanctioned quarantines have forced people to accept sudden changes in daily life. Moreover, those changes include high expectancies and fears associated with the massive vaccination. This has significantly affected emotional and social behavior (Holmes et al., 2020). Understanding human behavior in the presence of a global pandemic is crucial to the development of adequate strategies to cope with the social burden of the pandemic restrictions (Van Bavel et al., 2020). Historically, pandemics have created emotionally challenging situations for suitable behavioral responses toward the risk of being infected (Witte and Allen, 2000). Moreover, not all people develop the same behavioral responses toward a pandemic, leading to differences in risk perception, impact estimation, and responses to quarantine.

These responses may be partially determined by social cognitive skills and moral trends, together with being recognized as prosocial tendencies. Prosociality has been outlined with different theoretical frameworks. In this work, prosocial tendencies refer to psychological, cognitive, and affective processes that encourage beneficial effects on others (Clavien and Chapuisat, 2013; Clutton-Brock, 2009; Fehr and Fischbacher, 2003; Fehr et al., 2002) and produce positive and inclusive social interactions (Batson, 2011; Caprara et al., 2012). These tendencies and behaviors include helping others (Clavien and Chapuisat, 2013), reducing stress by comforting, sharing resources (Fehr and Fischbacher, 2003; Fehr et al., 2002), and supporting others' goals. Prosocial tendencies can also include actions that could be considered harmful in some contexts, such as rejection of and punishment for behaviors that affect others (Brethel-Haurwitz et al., 2016; Jensen, 2016; Sütterlin et al., 2011). Theoretically, those variables belong to two domains, social cognition, and moral cognition. Previous studies have shown that social cognition (Decety et al., 2016; N. Eisenberg et al., 2010; Telle and Pfister, 2016) and moral cognition (Nancy Eisenberg et al., 2013; Patrick et al., 2018; Turiel, 2015) are independent factors that can promote prosociality. The prosocial-related factors include different social cognition processes such as (a) empathy, which refers to the quality of being able to understand and share the feelings of others (Tania Singer and Klimecki, 2014; T. Singer et al., 2004); and (b) theory of mind (ToM), which conveys the skills to "mentalize" or infer others' mental states, emotions, and behaviors (BaronCohen, 2009; Baron-Cohen et al., 1985; Decety et al., 2016; Zaki et al., 2012); but also (c) moral behaviors and judgments that encourage social benefits, even if they call for personal or shared sacrifices (Kahane et al., 2018). Thus, prosocial-related factors, indexed by social and moral cognition profiles could affect compliance with pandemic-related regulations (Van Bavel et al., 2020; Witte and Allen, 2000).

Risk perception and acceptance of pandemic-related restrictions are socially rooted and promoted by prosocial behaviors (Dryhurst et al., 2020; Yang and Cho, 2017). For example, going out to work versus staying at home is not a personal choice during a pandemic, but a strong, socially impactful behavior. Prosociality is typically characterized by engagement in behavior that is intended to benefit others. It is measured by social and moral cognition (Jean Decety et al., 2016; Zaki et al., 2012). The factors associated with prosociality, then, indexed by empathy, ToM, and moral cognition could affect compliance with pandemic-related regulations (Van Bavel et al., 2020). Social cognition is critical to coping with uncertain and stressful scenarios (FeldmanHall et al., 2015). Empathy and ToM skills could impact appraisal of the risk of contracting the infection as well as the acceptance of public health warnings (Sharot, 2011). Equally, empathy skills can enhance our social sensitivity, triggering major personal distress and an overestimation of social information in the time of a pandemic (Tibi-Elhanany and Shamay-Tsoory, 2011; Zainal and Newman, 2018).

Moral tendencies (utilitarian vs. deontological) also impact the manner in which humans follow regulations in stressful social scenarios by activating either selfish or collaborative behaviors (Gino et al., 2016; Van Bavel et al., 2020). Specifically, instrumental harm situations (IH; situations that include acts that society usually forbids but became tolerable) and impartial beneficence situations (IB; situations that encourage contribution to the common good at the cost of personal sacrifice) modulate prosocial responses (Kahane et al., 2018). The relationship between these utilitarian and deontological responses and prosociality has been thoroughly studied (Capraro et al., 2018; Sunstein, 2005), however, their influence on behavioral responses to pandemic scenarios remains unknown.

Here we assessed the role that different factors associated with prosociality, including empathy, ToM, and moral behaviors, play in predicting risk estimation of infection consequences and acceptance of quarantine in pandemic times. Those behaviors have been conventionally studied when tracking population responses to socially threatening scenarios including the SARS-CoV2 pandemic and past epidemics (Clark et al., 2020; Han et al., 2021; Ibuka et al., 2010; Perrotta et al., 2021; Van Bavel et al., 2021). Through an open survey, we investigated the extent to which social (empathy and ToM) and moral cognition (IH and IB dilemmas) influence different crucial behaviors during pandemics. We were interested in assessing two significant COVID-19-related measures: (a) perceptions on COVID-19 consequences and (b) acceptance of quarantine with proven theoretical and empirical plausibility (AlSabbagh et al., 2021; Brooks et al., 2020; Manuell and Cukor, 2011; Pancani et al., 2021; Wang et al., 2021). First, we assessed the risk perception and the impact estimation of COVID-19, two measures tracking the capacity of perceiving and weighing the future consequences of SARS-CoV-2 (Han et al., 2021). Second, we assessed the acceptance of quarantine which is considered to be one of the most demanding public health requirements as quarantine involves, isolation, uncertainty, and negative effects on individuals mental and physical health (Brooks et al., 2020; Wang et al., 2021). Previous studies showed that individuals with enhanced affective sharing and deontological judgments overestimate social signals and generate worries and apprehensive expectative to social cues (Tibi-Elhanany and Shamay-Tsoory, 2011; Zainal and Newman, 2018). Based on these considerations, we hypothesized that individuals with higher scores in social cognition and deontologically oriented judgments would show increased risk perception, higher estimation of consequences of SARS-CoV-2, and at the same time, greater acceptance of quarantine.

\section{Methods}

Participants. The sample was comprised of 413 individuals. Participants were required to describe their gender with this prompt: "Please, write here the gender with which you have more identification". Our sample was composed of 314 women and 99 men from Colombia. The mean age of the sample was 23.46 $(\mathrm{SD}=9.76)$. On average, participants had 15.29 years of formal education $(\mathrm{SD}=3.28)$ and were middle class (estimated socioeconomic status, SES: $M=4.91 ; \quad \mathrm{SD}=1.40 ;$ scale $=1-8, \quad 1$ represents the lowest SES). No participants reported being infected with SARS-CoV-2.

Procedure. Participation was entirely voluntary; subjects accepted an invitation posted on the internet. All participants completed 
the entire survey and all experimental tasks $(M=25 \mathrm{~min})$. After, they reported age, sex, years of education, and SES. Furthermore, as control variables, participants reported depressive (using the Patient Health Questionnaire-9, (Kocalevent et al., 2013)) and anxiety symptoms (using the Generalized Anxiety Disorder questionnaire-7 (Löwe et al., 2008)). Upon completion of this assessment, subjects completed prosociality instruments further explained below.

Factors associated with prosociality. It comprises a set of cognitive skills and behaviors that promotes the benefit of others. Some frameworks assume prosociality to involve complex cognitive and behavioral processes (Jensen, 2016; Tania Singer and Klimecki, 2014), including social cognitive skills and moral behaviors that promote social benefits (Clutton-Brock, 2009). In our study, we assessed different factors associated with including a set of social cognition processes such as (a) empathy, (b) ToM, and a set of moral behaviors including responses in (c) intentional harm and (d) impartial beneficence situations.

Instruments for measuring factors associated with prosociality Empathy. Broadly refers to the quality of being able to share and understand the feelings (i.e. emotions, sensory states) of others (Tania Singer and Klimecki, 2014; T. Singer et al., 2004). This domain was assessed using a short version of the interpersonal reactivity index (IRI). This instrument measures dispositional empathy that considers empathy to encompass separate but related constructs, including affective (personal distress, empathic concern) and cognitive (perspective taking and fantasy) constructs (Davis, 1980; Ingoglia et al., 2016). Past studies have assessed domains of empathy by using IRI (Santamaría-García et al., 2017) in healthy individuals and patients with neuropsychiatric conditions (Dermody et al., 2016).

Theory of mind. The ability to mentalize and infer the mental states, emotions, and behaviors of others (Baron-Cohen, 2009; Baron-Cohen et al., 1985). This domain was assessed with the Reading the Mind in the Eyes Test (RMET) which evaluates emotional inference of ToM (Baron-Cohen, 2017; Baron-Cohen et al., 1985). This task consists of photographs of the ocular region of different faces. Participants must select the emotional state (in a group of four) that best describes the eye expression of each individual. In this study, we used a validated abridged version of RMET, consisting of 17 pictures (Olderbak et al., 2015). The total score in this task was indexed by the percentage of correct responses in selecting emotions.

Moral behaviors-instrumental harm. Behaviors include acts that society typically forbids but, eventually, become tolerable (Kahane et al., 2018). We assessed four histories with utilitarian moral dilemmas associated with SARS-CoV-2. Based on previous procedures (Kahane et al., 2018; Schein and Gray, 2018), participants answered three questions on $\mathrm{IH}$, including (a) to what extent they believed this action was wrong, using a scale of -5 (extremely bad) to 5 (extremely good); (b) to what extent they would behave in the same way as the protagonist of this situation, using a scale of 1 (low) to 10 (high); and (c) to what extent the action made by the protagonist of this situation had an intention to harm, using a scale of 1 (low) to 10 (high) (see Supplementary Information S1).

Moral behaviors-impartial beneficence. Behaviors that encourage contribution to the common good at the cost of personal sacrifice (Kahane et al., 2018). We developed four histories associated with SARS-CoV-2 that exposed situations promoting the greater good even at the expense of personal sacrifice. Participants answered a similar group of questions as described in IH (see Supplementary Information S2).

\section{Dependent SARS-CoV-2 measures}

Risk perception. Participants answered one question on their individual estimated percentage of risk (between $1 \%$ and $100 \%$ ) of contracting SARS-CoV-2 over the next year (see Supplementary Information S3).

Impact estimation. Participants answered six questions assessing medical (three questions) and social consequences (three questions) of virus-associated risks. A sum of positive answers on a total of six questions was used to build this variable (see Supplementary Information S3).

Acceptance of quarantine. Participants answered one question on their agreement with quarantine' requirements on a scale between 1 and 6 , in which 1 represented the lowest degree of acceptance of the quarantine.

Data analysis. We first ran multiple independent linear regressions for each dependent SARS-CoV-2 measure (risk perception, impact estimation, and acceptance of quarantine). We also implemented separate linear regression models for each independent prosocial predictor (social cognition and moral dilemmas). Regarding moral behaviors, we assessed three types of measures including moral judgment, harm detection, and acceptance of the behavior. All models included demographics (age, sex, years of education, and SES) and mood (depression and anxiety symptoms) as covariables (see Supplementary Information S4 for a further description of the analyzed variables).

In a subsequent analysis, we weighed the role of each group of independent variables in predicting dependent variables by running hierarchical regression models. Thus, we introduced each independent and control variable following a step-by-step procedure and determined the change of $R^{2}$ and $F$ measures due to each variable introduction. We employed $R^{2}$ and eta squared $\left(n^{2}\right)$ as effect size measures for significant effects. Furthermore, we reported for each regression model the standard errors and the expected effect sizes using a post hoc sensitivity analysis. Results showed that with an expected power of 0.95 and $\alpha$ level of 0.05 , the sample size we used was sufficient to detect a medium effect size $\left(n^{2}=0.13\right.$, critical $\left.t=1.98\right)$. We ran all statistical analyses using the JASP package version 0.14.1.

\section{Results}

A summary of the results is presented in Table 1 .

Risk perception. A model with social cognition, age, gender, years of education and depression, and anxiety variables as predictors and risk perception as a dependent variable reached significant values $\left[F(9,412)=8.77, p<0.001, R^{2}=0.19\right]$. Personal distress, empathic concern, and age were positively associated with risk perception. Particularly, higher levels of personal distress (beta $=0.14, t=2.84, p<0.001, \eta^{2}=0.18$ ), empathic concern (beta $=0.23, t=4.41, p<0.001, \eta^{2}=0.18$ ), and increased age (beta $=0.20, t=4.07, p<0.001, \eta^{2}=0.08$ ) were observed. Neither gender, years of education nor depression and anxiety variables reached significant values (see Table $2 \mathrm{~A}$ and Fig. 1).

A hierarchical regression model including only the social cognitive measures as predictors and risk perception as a dependent variable was significant $[F(11,401)=12.76, p<0.001$, $R^{2}=0.16$. A positive association was found between personal distress, empathic concern, and risk perception as higher personal distress (beta $=0.14, t=4.18, p<0.001, \eta^{2}=0.18$ ), and higher 


\begin{tabular}{|ll|}
\hline Table 1 Descriptive analysis of sociodemographic measures, \\
prosociality predictors (social and moral cognition), and \\
dependent measures. \\
Variables & $\begin{array}{l}\text { Mean values } \\
\text { Standard deviations }\end{array}$ \\
\hline Participants & $N=413$ \\
Sex (F:M) & $N=314: N=99$ \\
Age [mean (SD)] & $23.46(9.76)$ \\
Years of education (SD in years) & $15.29(3.29)$ \\
SES & $4.9(1.40)$ \\
Depressive symptoms (PHQ9) & $4.92(1.85)$ \\
Anxiety symptoms (GAD9) & $4.16(1.75)$ \\
Assessment of social cognition: Empathy IRI & $14.69(3.15)$ \\
Perspective taking & $13.42(3.88)$ \\
Fantasy & $10.65(3.29)$ \\
Personal distress & $16.46(2.69)$ \\
Empathic concern & \\
Assessment of social cognition: ToM (RMET) & \\
Reading the Mind in the Eyes Test (RMET) & $8.00(1.68)$ \\
Assessment of moral dilemmas: instrumental harm & \\
Moral judgment & $1.95(2.12)$ \\
Acceptance of character's behavior & $6.52(2.20)$ \\
Harm assessment & $4.15(2.09)$ \\
Assessment of moral dilemmas: Impartial beneficence & \\
Moral judgment & $2.36(1.47)$ \\
Acceptance of character's behavior & $6.88(1.63)$ \\
Assessment of dependent measures & $66.88(19.91)$ \\
Risk perception & $4.36(0.81)$ \\
Impact estimation & $5.53(0.81)$ \\
Acceptance of quarantine & \\
\hline
\end{tabular}

empathic concern (beta $=0.23, t=5.32, p<0.001, \eta^{2}=0.18$ ) significantly explained risk perception. Moreover, the hierarchical models that included the social cognitive measures plus control variables (age, gender, years of education, SES, depression and anxiety symptoms) in each step also reached significant values (see Table 2A).

We ran a second group of models with moral judgment, age, gender, years of education, depression and anxiety as independent factors and risk perception as a dependent variable. Results revealed that risk perception was also positively predicted by moral judgment measures $\left[F(9,412)=5.97, p<0.001, R^{2}=0.14\right]$. Particularly, judgments considering $\mathrm{IH}$ situations as harmful (beta $=0.15, \quad t=2.76, \quad p<0.01, \eta^{2}=0.14$ ) and increased age (beta $=0.24, t=4.67, p<0.001, \eta^{2}=0.08$ ), as well as gender (women: beta $=0.07, t=1.98, p<0.05, \eta^{2}=0.08$ ) were specific predictors of increased risk perception. No other analyses reached significant values (see Table 2B, Fig. 2).

A hierarchical regression model including only the moral measures as predictors and risk perception as a dependent measure was also significant and showed that moral judgments positively predicted risk perception $[F(11,412)=2.94, p<0.01]$. Particularly, (a) higher moral evaluation of IH situations (beta $\left.=0.27, t=2.44, p<0.01, \eta^{2}=0.14\right)$, (b) higher scores of harm in IH situations (beta $=0.14, t=1.99, p<0.05, \eta^{2}=0.10$ ), and (c) exhibiting increased acceptance of IB situations (beta $=0.14, t=1.98, p<0.05, \eta^{2}=0.10$ ) predicted higher risk

Table 2 Regression models of risk perception.

\begin{tabular}{|c|c|c|c|c|c|c|c|c|c|}
\hline Regression models & $F$ (model) & $R^{2}$ (model) & $P$ (model) & $\begin{array}{l}R^{2} \text { change } \\
\text { (model) }\end{array}$ & $\begin{array}{l}F \text { change } \\
\text { (model) }\end{array}$ & $\beta$ & $\begin{array}{l}\text { Standard errors } \\
\text { of factor }\end{array}$ & $t$ (factor) & $P$ (factor) \\
\hline \multicolumn{10}{|c|}{ (A) Risk perception regressed on Social cognitive measures } \\
\hline $\begin{array}{l}\text { Model with all variables } \\
\text { Hierarchical models }\end{array}$ & 8.77 & 0.20 & $<0.001^{\star}$ & 0.09 & 8.77 & & & & \\
\hline $\begin{array}{l}\text { Model } 1 \text { (only empathy and ToM } \\
\text { measures) }\end{array}$ & 12.76 & 0.16 & $<0.001^{\star}$ & 0.13 & 12.76 & & & & \\
\hline Personal distress & & & & & & 0.14 & 0.10 & 4.18 & $<0.01^{\star}$ \\
\hline Fantasy & & & & & & 0.06 & 0.08 & 1.31 & 0.19 \\
\hline RMET & & & & & & 0.03 & 0.18 & 0.76 & 0.44 \\
\hline Model 2 (Model $1+$ age) & 12.94 & 0.16 & $<0.001^{\star}$ & 0.13 & 12.94 & 0.20 & 0.03 & 3.40 & $<0.001^{\star}$ \\
\hline Model 3 (Model $1+$ gender) & 10.92 & 0.13 & $<0.001^{\star}$ & 0.12 & 12.14 & 0.06 & 0.72 & 1.36 & 0.17 \\
\hline Model 4 (Model 1 + education) & 10.38 & 0.13 & $<0.001^{\star}$ & 0.12 & 12.31 & 0.01 & 0.10 & 0.28 & 0.77 \\
\hline Model 5 (Model $1+$ SES) & 10.69 & 0.13 & $<0.001^{\star}$ & 0.12 & 12.69 & 0.04 & 0.22 & 0.99 & 0.32 \\
\hline $\begin{array}{l}\text { Model } 1 \text { (only moral behavior } \\
\text { measures) }\end{array}$ & 2.94 & 0.18 & $<0.01^{\star}$ & 0.03 & 2.94 & & & & \\
\hline IH Moral judgment & & & & & & 0.27 & 0.36 & 2.44 & $<0.05^{\star}$ \\
\hline $\mathrm{IH}$ Harm assessment & & & & & & 0.14 & 0.24 & 1.99 & $<0.05^{\star}$ \\
\hline IH Behavior acceptance & & & & & & 0.08 & 0.32 & 0.84 & 0.40 \\
\hline IB Moral judgment & & & & & & 0.10 & 0.29 & 1.72 & 0.07 \\
\hline IB Behavior acceptance & & & & & & 0.14 & 0.26 & 1.98 & $<0.05^{\star}$ \\
\hline Model 2 (Model $1+$ age) & 4.83 & 0.06 & $<0.001^{\star}$ & 0.03 & 3.41 & 0.17 & 0.03 & 3.72 & $<0.01^{\star}$ \\
\hline Model 3 (Model $1+$ gender) & 3.23 & 0.04 & $<0.05^{\star}$ & 0.03 & 3.02 & 0.10 & 0.78 & 2.14 & $<0.05^{\star}$ \\
\hline Model 4 (Model + education) & 1.91 & 0.03 & 0.08 & 0.03 & 1.91 & 0.05 & 0.10 & 0.96 & 0.33 \\
\hline Model 5 (Model $1+$ SES) & 1.98 & 0.03 & 0.08 & 0.03 & 1.99 & 0.03 & 0.23 & 0.75 & 0.45 \\
\hline Model 6 (Model $1+$ depression) & 2.99 & 0.06 & $<0.01^{\star}$ & 0.03 & 2.76 & 0.16 & 0.22 & 1.86 & 0.06 \\
\hline
\end{tabular}



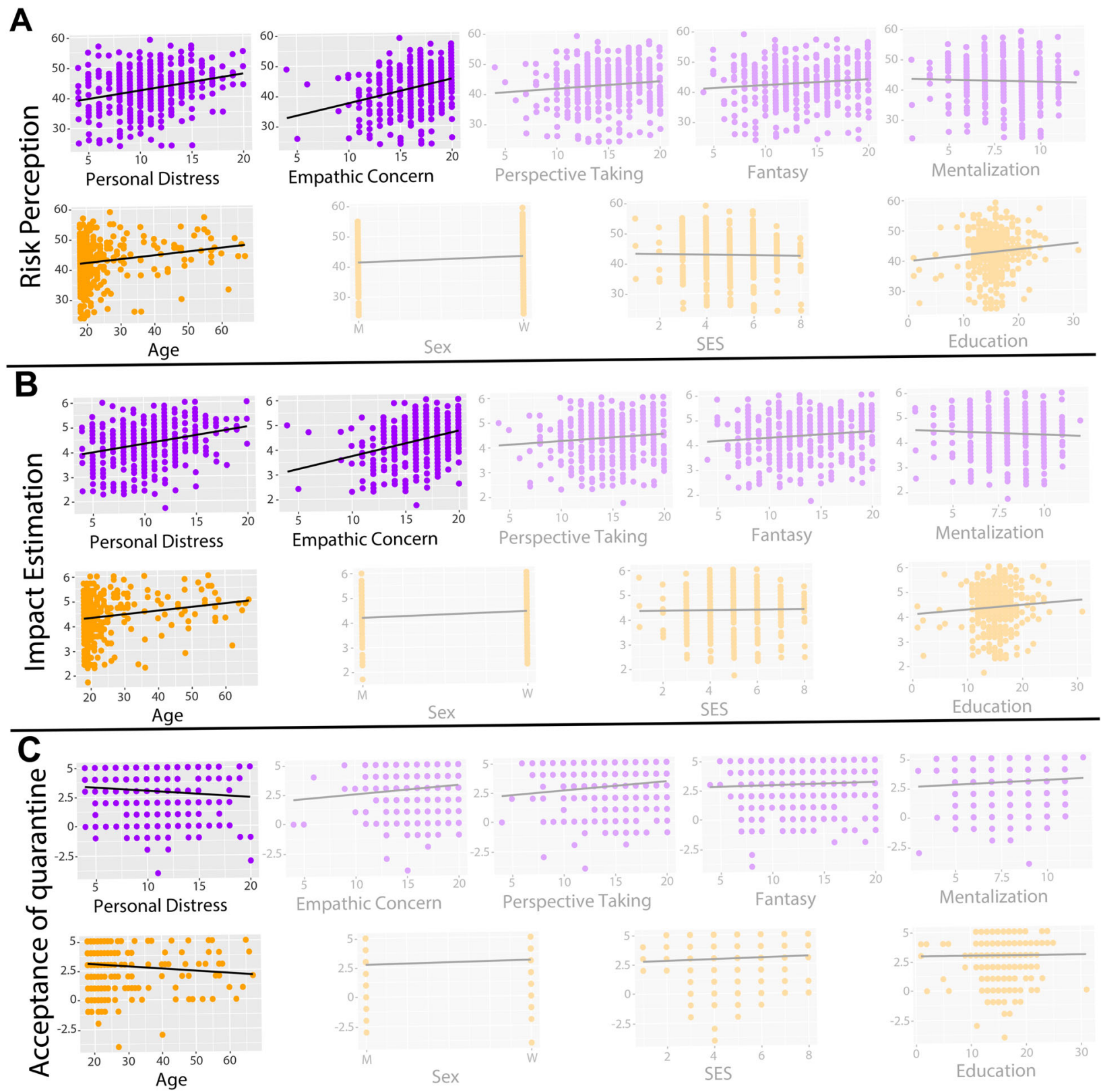

Fig. 1 Regression analyses result using social cognition measures as predictors. A Regression analysis using risk perception as the dependent variable. The dependent variable was explained by personal distress, empathic concern, and age. B Regression analysis using impact estimation as the dependent variable. This variable was predicted by personal distress, empathic concern, and age. C Regression analysis using the acceptance of quarantine as the dependent variable. This variable was predicted by personal distress, SES, and age. The non-significant variables were shaded across panels. SES socioeconomic status.

perception. The hierarchical models involving moral measures and age, gender, depression, and anxiety were also significant (see Table 2B).

Impact estimation. A model with social cognition, age, gender, years of education, depression and anxiety as predictors and impact estimation as the dependent variable was significant $[F(9$, $412)=9.35, p<0.0001, R^{2}=0.20$ ]. Impact estimation was positively predicted by personal distress (beta $=0.14, t=2.97$, $p<0.01, \eta^{2}=0.20$ ), empathic concern (beta $=0.25, t=4.73$, $p<0.001, \eta^{2}=0.22$ ), and increased age (beta $=0.21, t=4.18$, $\left.p<0.001, \eta^{2}=0.18\right)$. No other contrasts reached significant values (see Table 3 A, Fig. 1).
The hierarchical regression model with only the social cognitive measures and the same dependent variables was significant $\left[F(11,401)=14.22, p<0.001, R^{2}=0.14\right]$. Personal distress (beta $=0.20, t=4.73, p<0.001, \eta^{2}=0.22$ ) and empathic concern (beta $=0.29, t=5.61, p<0.001, \eta^{2}=0.22$ ) were positively associated with impact estimation. All hierarchical models combining social cognitive measures with control variables also reached significant values (see Table $3 \mathrm{~A}$ ).

A second group of models with moral judgment (and control variables) as predictors and impact estimation as a dependent variable also revealed significant values $[F(9,412)=5.87, p<0.001$, $\left.R^{2}=0.14\right]$. Increased harm detection in IH situations, (beta $=0.15$, $t=2.91, p<0.01, \eta^{2}=0.18$ ), increased age (beta $=0.24, t=4.72$, $p<0.001, \eta^{2}=0.16$ ), and sex (women: beta $=0.09, t=1.97$, 
A
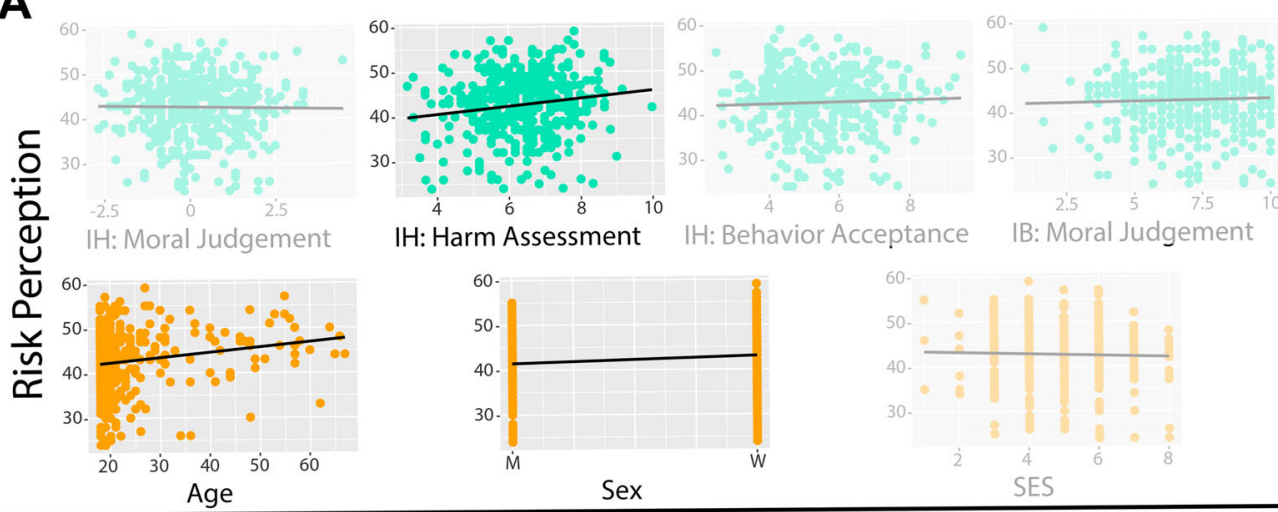

IH: Harm Assessment

$\mathrm{IH}$ : Behavior Acceptance

IB: Moral Judgement
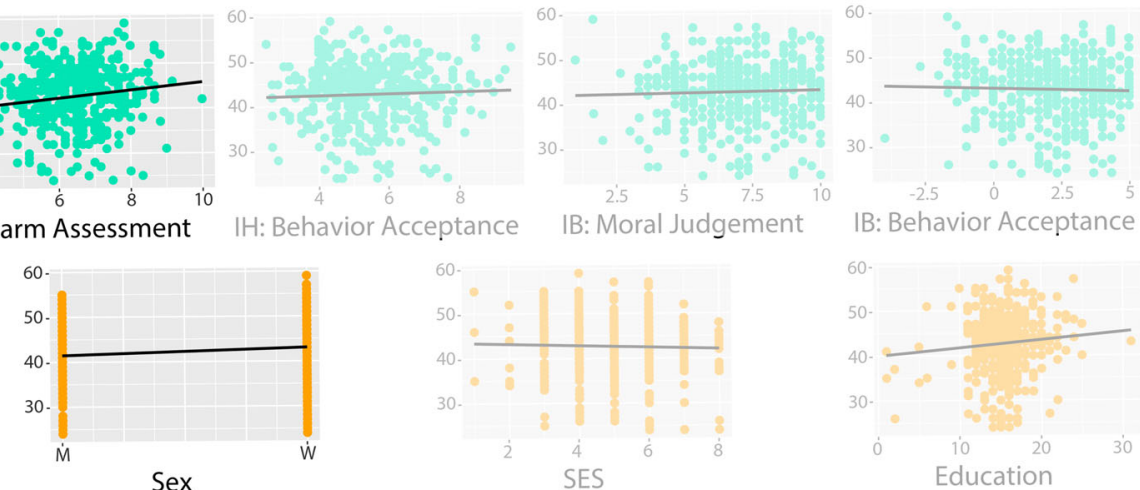

IB: Behavior Acceptance
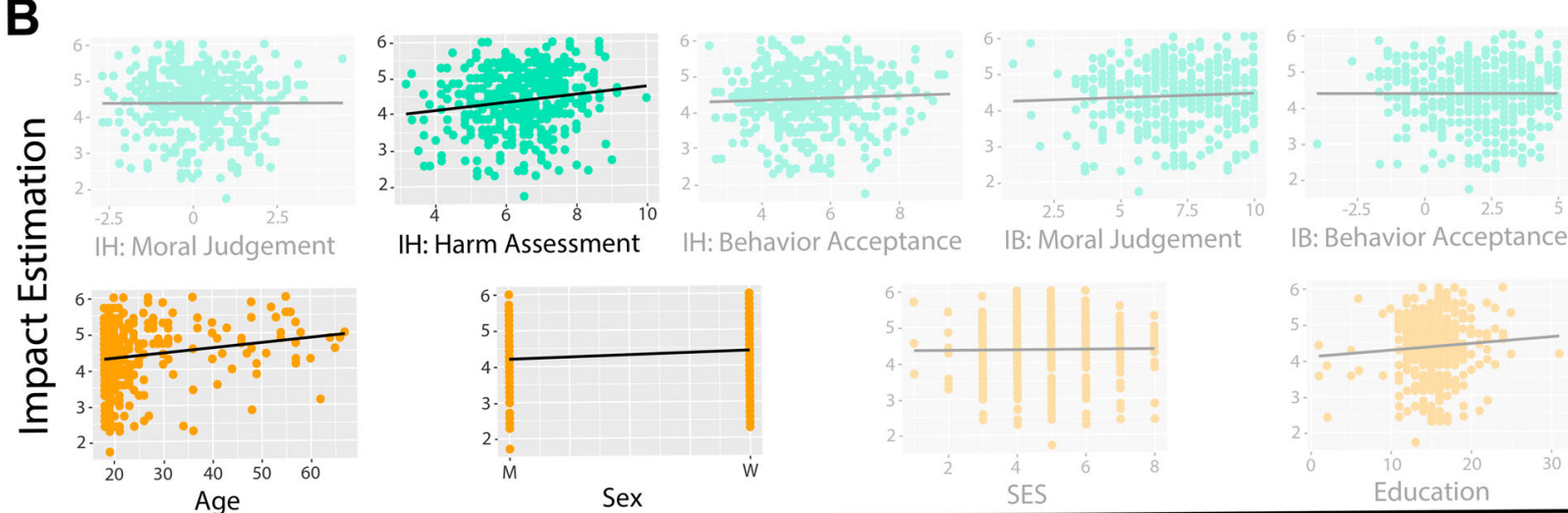

$\mathrm{IH}:$ Harm Assessment

IH: Behavior Acceptance

IB: Moral Judgement
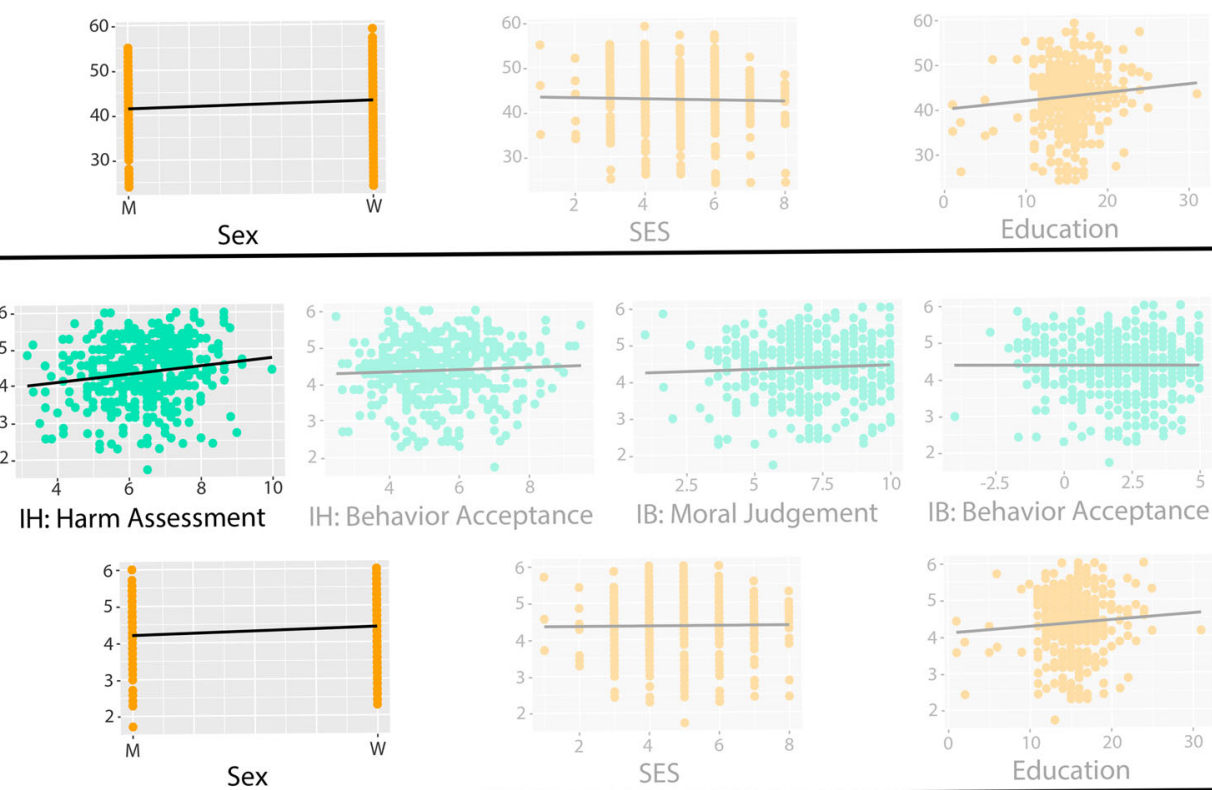

IB: Behavior Acceptance
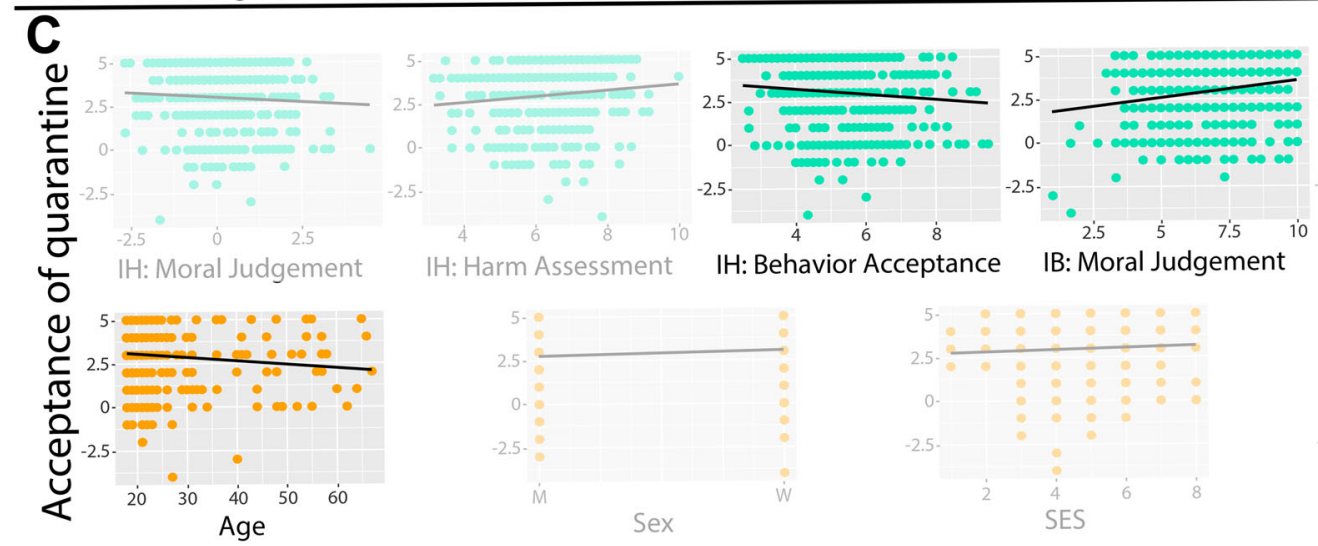

$\mathrm{IH}$ : Behavior Acceptance

IB: Moral Judgement
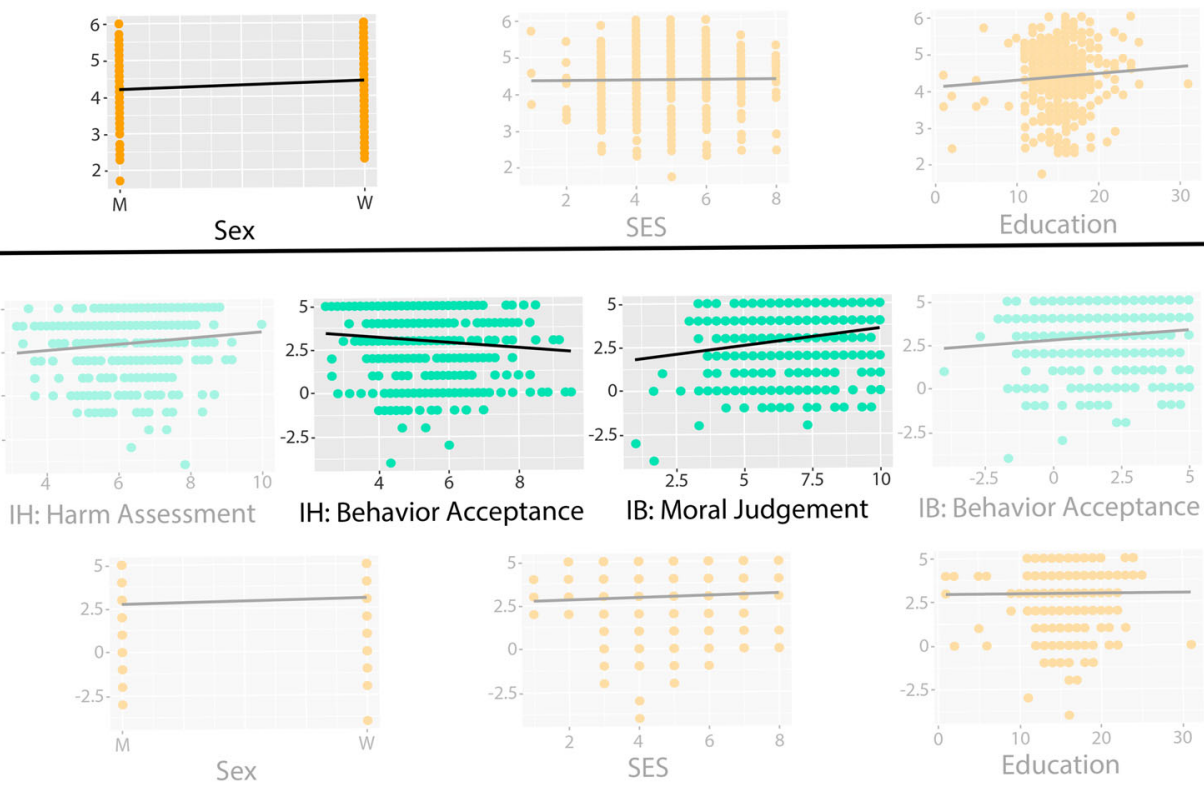

IB: Behavior Acceptance

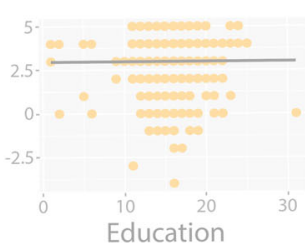

Fig. 2 Multiple regression analyses using moral judgment measures as predictors. A Regression analysis using risk perception as the dependent variable. This variable was explained by harm assessment in $\mathrm{IH}$, age, and sex. B Regression analysis using impact estimation as the dependent variable. This variable was predicted by harm assessment in $\mathrm{IH}$, age, and sex. C Regression analysis using the acceptance of quarantine as the dependent variable. This variable was predicted by increased acceptance of IB, reduced acceptance of $\mathrm{IH}$, and age. The non-significant variables were shaded across panels. IH instrumental harm, IB impartial beneficence, SES socioeconomic status.

$\left.p<0.05, \eta^{2}=0.12\right)$ predicted an amplified estimation of impact on personal life and society. No other analyses reached significant values.

A model with only the moral measures as predictors and impact estimation as a dependent variable reached significant values $\left[F(11,401)=3.39, p<0.01, R^{2}=0.06\right]$. The moral judgments positively predicted impact estimation as a higher moral judgment of $\mathrm{IH}$ situations (beta $=0.27, t=2.44, p<0.01$, $\eta^{2}=0.14$ ) and judgments considering $\mathrm{IH}$ as harmful (beta $=0.14$, $\left.t=1.99, p<0.05, \eta^{2}=0.10\right)$ better explained the impact estimation. Additionally, the hierarchical models of moral measures as well as age, gender, depression, and anxiety symptoms were also significant (see Table 3B, Fig. 2).
As a complementary analysis, we ran new regression models with two dependent variables of impact estimation: (a) medical impact estimation and (b) social impact estimation (see Supplementary Information S5). Results were consistent with those using combined scores.

Acceptance of quarantine. A model with social cognition and control variables as predictors and acceptance of quarantine as a dependent variable was significant $[F(9,412)=3.18, p<0.01$, $\left.R^{2}=0.09\right]$. The acceptance of quarantine was positively associated with personal distress (beta $=0.13, t=-2.45, p<0.01$, $\eta^{2}=0.18$ ) and negatively associated with age (beta $=-0.11$, 
Table 3 Regression models on impact estimation.

\begin{tabular}{|c|c|c|c|c|c|c|c|c|c|}
\hline Regression models & $F$ (model) & $R^{2}$ (model) & $P$ (model) & $\begin{array}{l}R^{2} \text { Change } \\
\text { (model) }\end{array}$ & $\begin{array}{l}F \text { Change } \\
\text { (model) }\end{array}$ & $\beta$ & $\begin{array}{l}\text { Standard } \\
\text { errors }\end{array}$ & $t$ & $\boldsymbol{p}$ \\
\hline \multicolumn{10}{|c|}{ (A) Impact estimation regressed on social cognitive measures } \\
\hline $\begin{array}{l}\text { Model with all variables included } \\
\text { Hierarchical models }\end{array}$ & 9.35 & 0.20 & $<0.001^{\star}$ & 0.09 & 9.35 & & & & \\
\hline $\begin{array}{l}\text { Model } 1 \text { (adding only empathy and } \\
\text { ToM measures) }\end{array}$ & 14.22 & 0.14 & $<0.001^{\star}$ & 0.14 & 14.22 & & & & \\
\hline Personal distress & & & & & & 0.20 & 0.10 & 4.26 & $<0.001^{\star}$ \\
\hline Fantasy & & & & & & 0.05 & 0.01 & 1.00 & 0.27 \\
\hline RMET & & & & & & 0.05 & 0.02 & 1.20 & 0.22 \\
\hline Model 2 (Model $1+$ age) & 14.22 & 0.17 & $<0.001^{\star}$ & 0.17 & 14.22 & 0.16 & 0.00 & 3.49 & $<0.001^{\star}$ \\
\hline Model 3 (Model $1+$ gender) & 12.21 & 0.15 & $<0.001^{\star}$ & 0.14 & 13.52 & 0.06 & 0.08 & 1.51 & 0.13 \\
\hline Model 4 (Model $1+$ education) & 11.92 & 0.15 & $<0.001^{\star}$ & 0.14 & 13.87 & 0.03 & 0.01 & 0.75 & 0.47 \\
\hline Model 5 (Model $1+$ SES) & 11.84 & 0.14 & $<0.001^{\star}$ & 0.14 & 14.02 & 0.01 & 0.02 & 0.06 & 0.94 \\
\hline $\begin{array}{l}\text { Model } 1 \text { (only moral behavior } \\
\text { measures) }\end{array}$ & 3.39 & 0.04 & $<0.001^{\star}$ & 0.04 & 3.39 & & & & \\
\hline IH Moral judgment & & & & & & 0.24 & 0.04 & 2.46 & $<0.05^{\star}$ \\
\hline $\mathrm{IH}$ harm assessment & & & & & & 0.16 & 0.04 & 3.11 & $<0.05^{\star}$ \\
\hline IH behavior acceptance & & & & & & 0.02 & 0.04 & 0.40 & 0.68 \\
\hline IB moral judgment & & & & & & 0.03 & 0.03 & 0.47 & 0.63 \\
\hline IB behavior acceptance & & & & & & 0.05 & 0.03 & 0.81 & 0.34 \\
\hline Model 2 (Model $1+$ age) & 5.06 & 0.07 & $<0.001^{\star}$ & 0.04 & 3.78 & 0.24 & 0.00 & 4.67 & $<0.01^{\star}$ \\
\hline Model 3 (Model $1+$ gender) & 3.72 & 0.05 & $<0.001^{\star}$ & 0.04 & 3.43 & 0.08 & 0.09 & 1.81 & 0.07 \\
\hline Model 4 (Model + education) & 3.27 & 0.04 & $<0.01^{\star}$ & 0.04 & 3.48 & 0.07 & 0.01 & 1.55 & 0.12 \\
\hline Model 5 (Model $1+$ SES) & 2.88 & 0.04 & $<0.01^{\star}$ & 0.04 & 3.45 & 0.01 & 0.03 & 0.59 & 0.54 \\
\hline Model 6 (Model $1+$ depression) & 5.67 & 0.07 & $<0.001^{\star}$ & 0.03 & 3.44 & 0.20 & 0.03 & 4.07 & $<0.05^{\star}$ \\
\hline
\end{tabular}

$\left.t=-1.99, p<0.05, \eta^{2}=0.14\right)$. No other analyses reached significant values (see Table 4A, Fig. 1).

A hierarchical regression model including only the social cognitive measures as predictors with the same dependent variable was significant $\left[F(11,401)=4.36, p<0.001, R^{2}=0.05\right]$. The model showed that higher personal distress (beta $=0.19$, $t=3.05, p<0.001, \eta^{2}=0.18$ ) and empathic concern (beta $=0.13$, $\left.t=2.31, p<0.01, \eta^{2}=0.18\right)$ significantly explained the acceptance of quarantine. All the hierarchical models that included social cognitive measures plus control variables following a stepby-step procedure also reached significant values (Table 4A).

A second group of models including moral judgment and control variables as predictors and acceptance of quarantine as a dependent variable also reached significance $[F(9,412)=3.01, p<0.01$, $\left.R^{2}=0.08\right]$. Major deontological tendencies in moral situations including a major agreement with IB situations (beta $=0.25$, $\left.t=3.34, p<0.001, \eta^{2}=0.16\right)$ and a reduced acceptance of behavior of $\mathrm{IH}$ situations (beta $=-0.13, t=-1.99, p<0.05, \eta^{2}=0.14$ ), as well as a reduced age (beta $=-0.22, t=-2.11, p<0.05, \eta^{2}=0.10$ ) predicted major acceptance of quarantine. No other contrasts reached significant values (see Fig. 2).

A hierarchical model with only moral judgment as a predictor and acceptance of quarantine as a dependent variable reached significant values $\left[F(11,401)=3.08, p<0.05, R^{2}=0.06\right]$. The acceptance of quarantine was positively predicted by deontological trends (higher behavior acceptance) in IB scenarios (beta $=0.23$, $\left.t=3.16, p<0.001, \eta^{2}=0.16\right)$. All hierarchical models of moral measures and control variables also reached significant values (Table 4B).

\section{Discussion}

This study shows that particular prosocial-related factors, indexed by empathy skills and moral deontological tendencies, predicted SARS-CoV-2 measures of risk, impact, and acceptance of quarantine. Notably, higher levels of personal distress and empathic concern (two core affective components of empathy (Melloni et al., 2013)) were associated with increased COVID-19 risk perception and impact estimation. Moreover, increased personal distress, a measure classically described as an aversive selforiented disposition to another's misfortune (H. Kim and Han, 2018), predicted a reduced acceptance of quarantine. More significant harm detection in instrumental harm scenarios predicted both higher risk perception and impact estimation regarding morality. Furthermore, in both moral situations (instrumental harm and impartial beneficence), deontological tendencies were associated with SARS-CoV measures. Increased acceptance of impartial beneficence and reduced acceptance of instrumental harm predicted better acceptance of quarantine. Results were preserved when only the social and moral predictors were considered and also when those analyses were performed with control variables (demographic and mental health variables). Other factors, including female gender identity and age (older individuals), also increased risk perception and impact estimation 
Table 4 Regression models on impact estimation.

\begin{tabular}{|c|c|c|c|c|c|c|c|c|c|}
\hline Hierarchical regression models & $\begin{array}{l}F \\
\text { (model) }\end{array}$ & $R^{2}$ (model) & $\begin{array}{l}P \\
\text { (model) }\end{array}$ & $\begin{array}{l}\text { R2 Change } \\
\text { (model) }\end{array}$ & $\begin{array}{l}F \text { Change } \\
\text { (model) }\end{array}$ & $\begin{array}{l}\text { Estimates } \\
\text { of factor }\end{array}$ & $\begin{array}{l}\text { Standard errors } \\
\text { of factor }\end{array}$ & $\begin{array}{l}t \text { (of } \\
\text { factor) }\end{array}$ & $\begin{array}{l}P \text { (of } \\
\text { factor) }\end{array}$ \\
\hline \multicolumn{10}{|c|}{ (A) Acceptance of quarantine regressed on social measures } \\
\hline $\begin{array}{l}\text { Model with all variables included } \\
\text { Hierarchical models }\end{array}$ & 3.18 & 0.09 & $<0.01^{\star}$ & 0.04 & 3.18 & & & & \\
\hline $\begin{array}{l}\text { Model } 1 \text { (adding only Empathy } \\
\text { and ToM measures) }\end{array}$ & 4.36 & 0.05 & $<0.001^{\star}$ & 0.05 & 4.36 & & & & \\
\hline Personal distress & & & & & & 0.19 & 0.01 & 3.60 & $<0.001^{\star}$ \\
\hline Fantasy & & & & & & 0.09 & 0.01 & 1.76 & 0.07 \\
\hline RMET & & & & & & 0.06 & 0.02 & 0.11 & 0.90 \\
\hline Model 2 (Model $1+$ age) & 3.71 & 0.05 & $<0.001^{\star}$ & 0.05 & 3.71 & 0.07 & 0.00 & 0.42 & 0.67 \\
\hline Model 3 (Model 1 + gender) & 4.30 & 0.06 & $<0.001^{\star}$ & 0.04 & 4.26 & 0.08 & 0.01 & 1.65 & 0.06 \\
\hline Model 4 (Model 1 + education) & 4.11 & 0.05 & $<0.001^{\star}$ & 0.04 & 4.19 & 0.07 & 0.03 & 1.45 & 0.14 \\
\hline Model 5 (Model $1+$ SES) & 4.03 & 0.05 & $<0.001^{\star}$ & 0.04 & 3.99 & 0.10 & 0.02 & 1.62 & 0.06 \\
\hline $\begin{array}{l}\text { Model } 1 \text { (only moral behavior } \\
\text { measures) }\end{array}$ & 3.08 & 0.04 & $<0.01^{\star}$ & 0.03 & 3.08 & & & & \\
\hline IH moral judgment & & & & & & 0.10 & 0.10 & 1.33 & 0.18 \\
\hline $\mathrm{IH}$ harm assessment & & & & & & 0.09 & 0.08 & 1.70 & 0.08 \\
\hline IH behavior acceptance & & & & & & 0.08 & 0.07 & 0.14 & 0.88 \\
\hline IB moral judgment & & & & & & 0.07 & 0.06 & 1.09 & 0.27 \\
\hline IB behavior acceptance & & & & & & 0.23 & 0.07 & 3.16 & $<0.01^{\star}$ \\
\hline Model 2 (Model 1+ age) & 3.39 & 0.21 & $<0.001^{\star}$ & 0.04 & 3.15 & 0.10 & 0.01 & 2.18 & $<0.05^{\star}$ \\
\hline Model 3 (Model 1 + gender) & 2.57 & 0.03 & $<0.05^{\star}$ & 0.03 & 3.02 & 0.00 & 0.18 & 0.12 & 0.89 \\
\hline Model 4 (Model + education) & 2.83 & 0.04 & $<0.05^{\star}$ & 0.04 & 3.24 & 0.06 & 0.02 & 1.24 & 0.21 \\
\hline Model 5 (Model $1+$ SES) & 2.57 & 0.03 & $<0.05^{\star}$ & 0.03 & 3.05 & 0.01 & 0.05 & 0.20 & 0.83 \\
\hline Model 6 (Model $1+$ depression) & 2.74 & 0.03 & $<0.05^{\star}$ & 0.03 & 3.00 & 0.05 & 0.04 & 1.02 & 0.30 \\
\hline
\end{tabular}

scores. Although the control factors modified the estimates of regression models, they did not impact the main results. Remarkably, our study revealed that both social measures and moral dilemmas reached a high convergence in predicting pandemic behaviors. The convergence between prosociality measures is supported by significant correlations between social cognitive skills and deontological judgments in IH and IB situations (see Supplementary Information S6). However, those measures are not subsumed by a unifying factor as revealed by confirmatory factor analyses (see Supplementary Information S7). As assumed in the theoretical background, two factors underlie these measures: a social cognitive factor (Empathy and ToM measures) and a moral judgment factor (responses in instrumental harm and impartial beneficence situations). Further studies assessing the interaction between prosociality and pandemic behaviors should include direct measures of prosociality aside from other related social and moral cognition measures.

Our results highlight the relevance of assessing social cognitive skills and moral dispositions related to prosocial behaviors, particularly in determining individual differences in the characterization of responses to social consequences of the pandemic.

Higher scores in the core and automatic affective empathy components (personal distress and empathic concern) predicted a greater COVID-19 risk perception and impact estimation. This pattern of results was consistent as revealed by similar findings upon analysis of the sub-measures of the impact estimation, including the medical impact estimation and the social impact independently. Our results suggest that affective components of empathy modulate public risk perception and adapt behavior to better suit the pandemic restrictions. Similar empathic responses to other stressful situations have been reported, including past respiratory epidemics and other public health threat situations (Cava et al., 2005; Lin and Margolin, 2014). Notably, individuals with a higher capacity to share affective experiences of others tend to exhibit more significant stress in socially challenging scenarios (Cristea et al., 2014). Our results also align with studies showing that personal distress and empathic concern can trigger fear and arousal symptoms generating worries, apprehensive expectations, and aversive behaviors to social information (Ferrer and Klein, 2015; Tibi-Elhanany and Shamay-Tsoory, 2011; Zainal and Newman, 2018). Thus, effective empathic components may act as amplifying lenses of common good threats by expanding the shared emotional experience to others.

High personal distress predicted reduced acceptance of quarantine. Personal distress is considered an aversive reaction (based on self-centered tendencies) to the emotional state of others $(\mathrm{H}$. Kim and Han, 2018). It is associated with maladaptive behaviors, including self-focused ruminative coping, neuroticism, self-criticism, and a broader trend of behaving in egoistic forms (H. Kim and Han, 2018; Tice et al., 2001). In this case, individuals with high personal distress may exhibit less acceptance of quarantine as an adaptive response to reduce the stress and self-oriented traits, including rumination, neuroticism, and negativism triggered by external impositions. Previous studies have reported a dissociation between empathetic characteristics such as personal distress skills and prosociality (H. Kim and Han, 2018; Tice et al., 
2001). In contrast to personal distress, prosociality does not involve aversive reactions to the suffering of others. Instead, it is characterized by feelings of warmth, concern, and care for the other and a solid motivation to improve the other's wellbeing (Böckler et al., 2016; Brethel-Haurwitz et al., 2016; Jensen, 2016; Tania Singer and Klimecki, 2014; Sütterlin et al., 2011). Individuals with higher personal distress tend to exhibit a reduced ability to behave cooperatively (H. Kim and Han, 2018; Tice et al., 2001). Prosociality, however, refers to the capacity to react to the benefit of others (Tania Singer and Klimecki, 2014).

Based on our results and previous reports, we suggest that the self-centered emotional reactions seen in individuals with high personal distress traits can promote resistance to social norms. Previous studies have shown that individuals with increased personal distress traits tend to exhibit a reduced capacity to behave cooperatively (Hortensius et al., 2016), exhibiting both reduced prosocial responses and higher physiological stress in the presence of others suffering (Hortensius et al., 2016; Tania Singer and Klimecki, 2014). In our report, individuals with personal distress tended to experience more stress associated with the current changes promoted by the pandemic. This avoidance effect could lead to increase self-protective responses.

Regarding moral behaviors, deontological choices (increased acceptance of impartial beneficence and an increased harm detection of instrumental harm scenarios), predicted SARS-CoV2 measures (risk perception, impact estimation, and acceptance of quarantine). Notably, the COVID-19 risk perception and impact estimation were predicted by increased harm detection in increased harm situations. Also, acceptance of quarantine was predicted by increased acceptance of self-sacrifices in impartial beneficence situations and a reduced acceptance in increased harm situations. Our results suggest an interplay between moral trends and risk perception (Böhm and Pfister, 2005; Holyoak and Powell, 2016). In particular, deontological tendencies have been associated with risk perception in agent-related situations (Böhm and Pfister, 2005). Similarly, in highly stressful situations, deontological tendencies manifest more apparently and help to anticipate future harmful consequences (Böhm and Pfister, 2005; Holyoak and Powell, 2016). Some deontological trends in moral situations, such as caring and fairness, are essential to predict behavioral compliance intentions for pandemic behaviors (Chan, 2021). Our results are consistent with these frameworks, as pandemic recommendations call for protecting oneself and others. The success of these prosocial-oriented recommendations depends on the community individuals who develop deontological behaviors (Chan, 2021).

The success of these prosocial-oriented recommendations depends on the community of individuals who develop deontological behaviors (Chan, 2021). Moreover, our results align with other recent studies showing that prosocial behaviors and traits predict the acceptance of pandemic requirements (CamposMercade et al., 2021). Prosocial behaviors measured before the pandemic also foreshadow acceptance of health requirements during the pandemic. Moreover, different psychological traits seem to predict pandemic responses. Among those traits, prosocial tendencies, neuroticism, and increased emotional reactivity determine agreement with health recommendations (Zettler et al., 2020). At the population level, regions that exhibited significant trust towards institutions and people tend to accept more readily the pandemic requirements than low-trust regions (Bargain and Aminjonov, 2020). Thus, in stressful situations such as the COVID-19 pandemic, assessing individual and group differences in social behaviors can be an efficient strategy to inform government behavioral insights.

Results also revealed that SARS-CoV-2 measures were modified by sociodemographic variables, including age and gender.
Both COVID-19 risk perception and impact estimation were associated with increased age. Paradoxically, older people exhibited minor acceptance of quarantine. A possible explanation for this pattern of results would be that older people are more cautious and risk-averse in the health domain but less compliant with social impositions (Bonem et al., 2015). Minimal acceptance of quarantine observed in older people could also be related to health system prohibitions as more significant restrictions are imposed on older people than younger people. Second, our results revealed that female gender identity predicted increased COVID19 risk perception and impact estimation when this factor was assessed in models of moral judgment. This pattern of results coincides with previous studies showing gender differences in utilitarian predispositions (Baez et al., 2017). In our results, moral trends were tracked by pandemic-related situations. This type of moral scenario could increase stress and apprehensive expectations in women who have shown to be more affected than men by behavior risks associated with pandemics.

Moreover, our results align with previous studies showing gender differences in moral dilemma judgments are due to differences in affective responses to harm rather than cognitive evaluations of outcomes (Baez et al., 2017; Friesdorf et al., 2015). Gender roles seem to be highly determinant of risk perception (Harris and Jenkins, 2006) and estimation of social threats (Friesdorf et al., 2015). Our results also convey novel evidence suggesting that gender determines risk perception and impact estimation when moral trends are considered, but no acceptance of quarantine. Although women tend to exhibit significant stress in socially challenging situations (Baez et al., 2017), we did not observe gender effects in the social cognition model in our study. Crucially, the absence of gender interactions with social cognitionpandemic behaviors could be explained by self-administered social cognitive measures. Past studies have revealed controversial gender results in self-administered tasks of social cognition (Baez et al., 2017; Löffler and Greitemeyer, 2021). More research is needed to clarify better the role of gender in social cognition predispositions related to pandemic responses.

Furthermore, the COVID-19 pandemic has mainly been associated with depression and anxiety symptoms (Pan et al., 2021; Riello et al., 2020). In our study, however, those symptoms were not associated with the pandemic behaviors. Thus, the measures associated with prosociality and the pandemic behaviors seem to be related irrespective of the expected presence of anxiety and depression symptoms observed in the pandemic.

These overall results point to multimodal but non-homogenous effects of prosociality. More robust effects of social cognition were observed than moral cognition, as shown by effect sizes. Empathy may guide responses more straightforwardly, given that it seems to be a more stable trait mark of personality than morality choices, which are modified by different situations (Decety and Cowell, 2014). Our results confirm recent reports showing that empathy predicts the motivation to adhere to physical distancing and wearing face masks (Pfattheicher et al., 2020). Inducing empathy also promotes the motivation to stick to these measures (Pfattheicher et al., 2020). However, our results provide a specific characterization of empathy and moral process as relevant factors associated with pandemic responses.

Results underscore the selective influence of affective empathy (over cognitive empathy components and ToM) in modulating pandemic behaviors. Increased affective empathy leads to the overvaluation of social cues in stressful situations and promotes prosociality (Zaki et al., 2012). Furthermore, our study also adds new evidence on the role of deontological tendencies on pandemic responses. In our research, the moral behaviors shown to impact all of the pandemic-related behaviors and seem to be contingent on the type of situation, distress level, contextual 
information, and gender influences (Baez et al., 2017; Holyoak and Powell, 2016). These results provide basic knowledge of at least some components to be identified to promote better compliance with adequate responses to the pandemic.

Our study has some limitations that call for further research. First, our sample was obtained from Colombian participants; these results should not be generalized to other populations. Colombia, however, has so far exhibited a highly inequitable distribution of COVID-19 vaccines-a trend seen in other countries of the Global South. Currently, only $6.5 \%$ of the Colombian population has been fully vaccinated. This means that new variants are likely to emerge, and loss of life-alongside many other harmful impacts-of the most vulnerable will continue. In this context, the design of behavioral studies and interventions aimed to identify the main prepositional factors associated with behavioral change and adequate responses to pandemic restrictions are critically needed. Our sample was recruited by convenience, collected via an online survey, and was mainly represented by a population of educated Colombian people with access to virtual resources and the internet. The mentioned factors may limit sample representativeness, statistical inferences, and the generalizability of findings to some degree (Shi et al., 2020). Nevertheless, our sampling was recruited virtually considering pandemic restrictions and coincided with other studies assessing behaviors during pandemics (Imbriano et al., 2021; Perrotta et al., 2021). Although the sample size in our study was supported by power analysis and previous studies assessing pandemic behavior (Dryhurst et al., 2020), future studies should determine behavior by using large, globally collected, and probabilistic sample sizes. This also may allow for the ability to test the stability, generalizability, and specificity of results across different cultural backgrounds.

Second, our sample was mainly represented by females. This female overrepresentation agrees with previous studies showing that women are more willing to participate in online surveys (Smith, 2008). We found that gender impacts participants' pattern of responses, but female overrepresentation may bias the results. However, gender was only significant in two of the six regression models used to assess risk perception, impact estimation, and acceptance of quarantine. The gender effects observed in the moral models are consistent with previous studies (Baez et al., 2017). In our study, moral trends were tracked by pandemicrelated situations, which could increase women's stress; women have been shown to be more affected than men by behavior challenges and contagion risks associated with pandemics. In any case, future studies should assess the role of social and moral cognitive domains in predicting pandemic behaviors by using gender-balanced sampling. Moreover, new studies could evaluate the extent to which stress levels and anxiety traits interact with gender in predicting pandemic behaviors.

Third, another limitation of our study is the composition of our sample-mainly younger adults. Previous studies have revealed that younger adults seem to exhibit less compliance with pandemic restrictions (Kim and Crimmins, 2020). In our study, younger adults showed different levels of social cognition and moral behaviors than older adults and reached higher scores in empathy scales (Grühn et al. 2008) but tended to show more utilitarian responses in moral dilemmas (McNair et al., 2019). Thus, our results could be affected by the mentioned differences and reveal mainly the pattern of interactions between prosociality measures and pandemic behaviors in younger adults. Future studies should assess the interactions between measures associated with prosociality and pandemic behaviors across the lifespan and in different stages of the pandemic.

Fourth, although we based our assessments on standard instruments reported in previous research, we used self-reported tools with an unbalanced number of items across measures to track prosocial tendencies. Self-report measures are subject to biases and limitations, as are apparent in other studies of prosocial tendencies (Böckler et al., 2016; Han et al., 2021; Ibuka et al., 2010) including studies on the COVID-19 pandemic (Dinić and Bodroža, 2021; Van Bavel et al., 2021). Future studies should implement prosocial, social, and moral cognition assessments combining subjective, objective, and real-life measurements. Additional research on prosocial-related standards is urgently required in the current vaccination stage, especially considering regional inequalities.

Finally, although the COVID-19 related measures employed in this report are considered critical by current theoretical developments (Van Bavel et al., 2020), the role of prosocial tendencies in predicting other significant pandemic behaviors should be further assessed. Future studies should parse other pandemic-related behaviors such as acceptance of medical requirements, vaccination, and behavioral change after experiences of negative COVID19 health consequences. Moreover, future research should combine self-report and implicit measures to accurately weigh pandemic responses. Furthermore, our study, similar to others in the field (Pfattheicher et al., 2020; Van Bavel et al., 2021; van de Groep et al., 2020), does not provide control for the medical impacts of COVID-19 that could bias the responses. In spite of this, results presented by this study are controlled for the effects of different sociodemographic and mental health confounds which are often unreported in previous related studies. Future research should assess the effect of the COVID-related health outcomes on the behavioral responses during the pandemic.

In summary, our results revealed the extent to which two sides of a group of prosocial-related factors impact pandemic behaviors. On the one hand, empathy skills and deontological choices predicted acceptance of pandemic requirements (acceptance of quarantine). However, these traits also triggered the overestimation of risk signals associated with infection (risk perception and impact estimation). Integrating social information may be crucial to track pandemic risks, as previously reported in other types of threats (Dryhurst et al., 2020; Kasperson et al., 1988). The factors associated with prosociality are adaptive and evolving traits that can follow risks, impacts, and compliance norms in scenarios presenting uncertainty. We hope our results can inform the development of strategies to increase public health compliance by appealing to prosociality tendencies (as opposed to maximizing restrictions and punishments) to face the social challenges associated with the pandemic in Colombia. Current results are now even more relevant in the context of Global South vaccine inequalities and delayed responses to massive vaccination in Colombia. However, our results must be considered with caution, as the study reports a non-randomized population from a single country during the pandemic lockdown. Thus, it is necessary to assess in new studies to what extent these results can develop statistical inferences about the role of social and moral cognition in modulating pandemic behaviors in other regions of the world.

\section{Data availability}

The data that support the findings of this study are available from the corresponding author upon reasonable request.

Received: 12 June 2021; Accepted: 20 December 2021; Published online: 07 January 2022

\section{References}

Al-Sabbagh MQ, Al-Ani A, Mafrachi B, Siyam A, Isleem U, Massad FI, Abufaraj M (2021) Predictors of adherence with home quarantine during COVID-19 
crisis: the case of health belief model. Psychol Health Med (1):1-13. https:// doi.org/10.1080/13548506.2021.1871770

Baez S, Flichtentrei D, Prats M, Mastandueno R, García AM, Cetkovich M, Ibáñez A (2017) Men, women... who cares? A population-based study on sex differences and gender roles in empathy and moral cognition. PLoS ONE 12(6):e0179336. https://doi.org/10.1371/journal.pone.0179336

Bargain O, Aminjonov U (2020) Trust and compliance to public health policies in times of COVID-19. J Public Econ 192:104316. https://doi.org/10.1016/ j.jpubeco.2020.104316

Baron-Cohen S (2009) Autism: the empathizing-systemizing (E-S) theory. Ann NY Acad Sci 1156:68-80. https://doi.org/10.1111/j.1749-6632.2009.04467.x

Baron-Cohen S (2017) The eyes as window to the mind. Am J Psychiatry 174(1):1-2. https://doi.org/10.1176/appi.ajp.2016.16101188

Baron-Cohen S, Leslie AM, Frith U (1985) Does the autistic child have a "theory of mind"? Cognition 21(1):37-46. https://doi.org/10.1016/0010-0277(85)90022-8

Batson CD (2011) Altruism in humans. Oxford University Press, Oxford

Böckler A, Tusche A, Singer T (2016) The structure of human prosociality: differentiating altruistically motivated, norm motivated, strategically motivated, and self-reported prosocial behavior. Soc Psychol Personal Sci 7(6):530-541

Böhm G, Pfister HR (2005) Consequences, morality, and time in environmental risk evaluation. J Risk Res 8(6):461-479

Bonem EM, Ellsworth PC, Gonzalez R (2015) Age differences in risk: perceptions, intentions and domains. J Behav Decision Mak 28(4):317-330

Brethel-Haurwitz KM, Stoycos SA, Cardinale EM, Huebner B, Marsh AA (2016) Is costly punishment altruistic? Exploring rejection of unfair offers in the Ultimatum Game in real-world altruists. Sci Rep 6. https://doi.org/10.1038/srep18974

Brooks SK, Webster RK, Smith LE, Woodland L, Wessely S, Greenberg N, Rubin GJ (2020) The psychological impact of quarantine and how to reduce it: rapid review of the evidence. The Lancet 395(10227):912-920

Campos-Mercade P, Meier AN, Schneider FH, Wengström E (2021) Prosociality predicts health behaviors during the COVID-19 pandemic. J Public Econ 195:104367. https://doi.org/10.1016/j.jpubeco.2021.104367

Caprara GV, Alessandri G, Eisenberg N (2012) Prosociality: the contribution of traits, values, and self-efficacy beliefs. J Pers Soc Psychol 102(6):1289-1303. https://doi.org/10.1037/a0025626

Capraro V, Sippel J, Zhao B, Hornischer L, Savary M, Terzopoulou Z, Griffioen SF (2018) People making deontological judgments in the Trapdoor dilemma are perceived to be more prosocial in economic games than they actually are. PLoS ONE 13(10):e0205066

Cava MA, Fay KE, Beanlands HJ, McCay EA, Wignall R (2005) Risk perception and compliance with quarantine during the SARS outbreak. J Nurs Scholarsh 37(4):343-347. https://doi.org/10.1111/j.1547-5069.2005.00059.x

Chan EY (2021) Moral foundations underlying behavioral compliance during the COVID-19 pandemic. Pers Individ Dif 171:110463. https://doi.org/10.1016/ j.paid.2020.110463

Clark C, Davila A, Regis M, Kraus S (2020) Predictors of COVID-19 voluntary compliance behaviors: An international investigation. Global Transit 2:76-82. https://doi.org/10.1016/j.glt.2020.06.003

Clavien C, Chapuisat M (2013) Altruism across disciplines: one word, multiple meanings. Biol Philos 28(1):125-140

Clutton-Brock T (2009) Cooperation between non-kin in animal societies. Nature 462(7269):51-57

Cristea IA, Legge E, Prosperi M, Guazzelli M, David D, Gentili C (2014) Moderating effects of empathic concern and personal distress on the emotional reactions of disaster volunteers. Disasters 38(4):740-752. https://doi.org/10.1111/disa.12075

Davis MH (1980) Interpersonal reactivity index. Edwin Mellen Press

Decety J, Bartal IB, Uzefovsky F, Knafo-Noam A (2016) Empathy as a driver of prosocial behaviour: highly conserved neurobehavioural mechanisms across species. Philos Trans R Soc Lond B Biol Sci 371(1686):20150077. https:// doi.org/10.1098/rstb.2015.0077

Decety J, Bartal IB-A, Uzefovsky F, Knafo-Noam A (2016) Empathy as a driver of prosocial behaviour: highly conserved neurobehavioural mechanisms across species. Philos Trans R Soc B: Biol Sci 371(1686):20150077

Decety J, Cowell JM (2014) Friends or foes: is empathy necessary for moral behavior? Perspect Psychol Sci 9(5):525-537

Dermody N, Wong S, Ahmed R, Piguet O, Hodges JR, Irish M (2016) Uncovering the neural bases of cognitive and affective empathy deficits in Alzheimer's disease and the behavioral-variant of frontotemporal dementia. J Alzheimers Dis 53(3):801-816. https://doi.org/10.3233/jad-160175

Dinić BM, Bodroža B (2021) COVID-19 protective behaviors are forms of prosocial and unselfish behaviors. Front Psychol 12(1128) https://doi.org/ 10.3389/fpsyg.2021.647710

Dryhurst S, Schneider CR, Kerr J, Freeman AL, Recchia G, Van Der Bles AM, van der Linden S (2020) Risk perceptions of COVID-19 around the world. J Risk Res 1-13

Eisenberg N, Eggum ND, Di Giunta L (2010) Empathy-related responding: associations with prosocial behavior, aggression, and intergroup relations. Soc Issues Policy Rev 4(1):143-180. https://doi.org/10.1111/j.1751-2409.2010.01020.x
Eisenberg N, Morris, AS (2013) Moral cognitions and prosocial responding in adolescence. In handbook of adolescent psychology: second ed. (pp. 155-188). wiley. https://doi.org/10.1002/9780471726746

Fehr E, Fischbacher U (2003) The nature of human altruism. Nature 425(6960):785-791

Fehr E, Fischbacher U, Gächter S (2002) Strong reciprocity, human cooperation, and the enforcement of social norms. Hum Nat 13(1):1-25. https://doi.org/ 10.1007/s12110-002-1012-7

FeldmanHall O, Raio CM, Kubota JT, Seiler MG, Phelps EA (2015) The effects of social context and acute stress on decision making under uncertainty. Psychol Sci 26(12):1918-1926. https://doi.org/10.1177/0956797615605807

Ferrer RA, Klein WM (2015) Risk perceptions and health behavior. Curr Opinion Psychol 5:85-89

Friesdorf R, Conway P, Gawronski B (2015) Gender differences in responses to moral dilemmas: a process dissociation analysis. Personal Soc Psychol Bull 41(5):696-713

Gino F, Norton MI, Weber RA (2016) Motivated Bayesians: feeling moral while acting egoistically. J Econ Perspect 30(3):189-212

Grühn D, Rebucal K, Diehl M, Lumley M, Labouvie-Vief G (2008) Empathy across the adult lifespan: longitudinal and experience-sampling findings. Emotion 8(6):753-765. https://doi.org/10.1037/a0014123

Han Q, Zheng B, Agostini M, Bélanger JJ, Gützkow B, Kreienkamp J, Leander NP (2021) Associations of risk perception of COVID-19 with emotion and mental health during the pandemic. J Affect Disord 284:247-255. https:// doi.org/10.1016/j.jad.2021.01.049

Harris CR, Jenkins M, Glaser D (2006) Gender differences in risk assessment: Why do women take fewer risks than men? Judgment Decis Mak 1(1):48-63

Holmes EA, O'Connor RC, Perry VH, Tracey I, Wessely S, Arseneault L, Bullmore E (2020) Multidisciplinary research priorities for the COVID-19 pandemic: a call for action for mental health science. Lancet Psychiatry 7(6):547-560

Holyoak KJ, Powell D (2016) Deontological coherence: a framework for commonsense moral reasoning. Psychol Bull 142(11):1179

Hortensius R, Schutter DJLG, de Gelder B (2016) Personal distress and the influence of bystanders on responding to an emergency. Cogn Affect Behav Neurosci 16(4):672-688. https://doi.org/10.3758/s13415-016-0423-6

Ibuka Y, Chapman GB, Meyers LA, Li M, Galvani AP (2010) The dynamics of risk perceptions and precautionary behavior in response to 2009 (H1N1) pandemic influenza. BMC Infect Dis 10(1):296. https://doi.org/10.1186/14712334-10-296

Imbriano G, Larsen EM, Mackin DM, An AK, Luhmann CC, Mohanty A, Jin J (2021) Online survey of the impact of COVID-19 risk and cost estimates on worry and health behavior compliance in young adults. Front Public Health 9(157) https://doi.org/10.3389/fpubh.2021.612725

Ingoglia S, Lo Coco A, Albiero P (2016) Development of a brief form of the interpersonal reactivity index (B-IRI). J Pers Assess 98(5):461-471. https:// doi.org/10.1080/00223891.2016.1149858

Jensen K (2016) Prosociality. Curr Biol 26(16):R748-R752. https://doi.org/10.1016/ j.cub.2016.07.025

Kahane G, Everett JAC, Earp BD, Caviola L, Faber NS, Crockett MI, Savulescu J (2018) Beyond sacrificial harm: a two-dimensional model of utilitarian psychology. Psychol Rev 125(2):131-164. https://doi.org/10.1037/rev0000093

Kasperson RE, Renn O, Slovic P, Brown HS, Emel J, Goble R, Ratick S (1988) The social amplification of risk: a conceptual framework. Risk analysis 8(2):177-187

Kim H, Han S (2018) Does personal distress enhance empathic interaction or block it? Personal Individ Differ 124:77-83. https://doi.org/10.1016/j.paid.2017.12.005

Kim JK, Crimmins EM (2020) How does age affect personal and social reactions to COVID-19: Results from the national Understanding America Study. PLoS ONE 15(11):e0241950

Kocalevent R-D, Hinz A, Brähler E (2013) Standardization of the depression screener patient health questionnaire (PHQ-9) in the general population. General Hosp Psychiatry 35(5):551-555

Lin Y-R, Margolin D (2014) The ripple of fear, sympathy and solidarity during the Boston bombings. EPJ Data Sci 3(1):31

Löffler CS, Greitemeyer T (2021) Are women the more empathetic gender? The effects of gender role expectations. Curr Psychol https://doi.org/10.1007/ s12144-020-01260-8

Löwe B, Decker O, Müller S, Brähler E, Schellberg D, Herzog W, Herzberg PY (2008) Validation and standardization of the Generalized Anxiety Disorder Screener (GAD-7) in the general population. Med care 1(1):266-274

Manuell ME, Cukor J (2011) Mother Nature versus human nature: public compliance with evacuation and quarantine. Disasters 35(2):417-442

McNair S, Okan Y, Hadjichristidis C, de Bruin WB (2019) Age differences in moral judgment: older adults are more deontological than younger adults. J Behav Decision Mak 32(1):47-60

Melloni M, Lopez V, Ibanez A (2013) Empathy and contextual social cognition. Cogn Affect Behav Neurosci https://doi.org/10.3758/s13415-013-0205-3

Olderbak S, Wilhelm O, Olaru G, Geiger M, Brenneman MW, Roberts RD (2015) A psychometric analysis of the reading the mind in the eyes test: toward a 
brief form for research and applied settings. Front Psychol 6:1503. https:// doi.org/10.3389/fpsyg.2015.01503

Pan K-Y, Kok AAL, Eikelenboom M, Horsfall M, Jörg F, Luteijn RA, Penninx BWJH (2021) The mental health impact of the COVID-19 pandemic on people with and without depressive, anxiety, or obsessive-compulsive disorders: a longitudinal study of three Dutch case-control cohorts. The Lancet Psychiatry 8(2):121-129. https://doi.org/10.1016/S2215-0366(20)30491-0

Pancani L, Marinucci M, Aureli N, Riva P (2021) Forced social isolation and mental health: A study on 1,006 italians under covid-19 lockdown. Front Psychol (12) 1540

Patrick RB, Bodine AJ, Gibbs JC, Basinger KS (2018) What accounts for prosocial behavior? Roles of moral identity, moral judgment, and self-efficacy beliefs. J Genet Psychol 179(5):231-245. https://doi.org/10.1080/00221325.2018.1491472

Perrotta D, Grow A, Rampazzo F, Cimentada J, Del Fava E, Gil-Clavel S, Zagheni E (2021) Behaviours and attitudes in response to the COVID-19 pandemic: insights from a cross-national Facebook survey. EPJ Data Sci 10(1):17. https://doi.org/10.1140/epjds/s13688-021-00270-1

Pfattheicher S, Nockur L, Böhm R, Sassenrath C, Petersen MB (2020) The emotional path to action: empathy promotes physical distancing and wearing of face masks during the COVID-19 pandemic. Psychol Sci 31(11):1363-1373

Riello M, Purgato M, Bove C, MacTaggart D, Rusconi E (2020) Prevalence of posttraumatic symptomatology and anxiety among residential nursing and care home workers following the first COVID-19 outbreak in Northern Italy. R Soc Open Sci 7(9):200880. https://doi.org/10.1098/rsos.200880

Santamaría-García H, Baez S, García AM, Flichtentrei D, Prats M, Mastandueno R, Ibáñez A (2017) Empathy for others' suffering and its mediators in mental health professionals. Sci Rep 7(1):6391. https://doi.org/10.1038/s41598-017-06775-y

Schein C, Gray K (2018) The theory of dyadic morality: reinventing moral judgment by redefining harm. Pers Soc Psychol Rev 22(1):32-70. https://doi.org/ $10.1177 / 1088868317698288$

Sharot T (2011) The optimism bias. Curr Biol 21(23):R941-R945

Shi L, Lu Z-A, Que J-Y, Huang X-L, Liu L, Ran M-S, Sun Y-K (2020) Prevalence of and risk factors associated with mental health symptoms among the general population in China during the coronavirus disease 2019 pandemic. JAMA Network Open 3(7):e2014053-e2014053

Singer T, Klimecki OM (2014) Empathy and compassion. Curr Biol 24(18):R875-R878

Singer T, Seymour B, O'Doherty J, Kaube H, Dolan RJ, Frith CD (2004) Empathy for pain involves the affective but not sensory components of pain. Science 303(5661):1157-1162. https://doi.org/10.1126/science.1093535

Smith G (2008) Does gender influence online survey participation?: a recordlinkage analysis of university faculty online survey response behavior. ERIC Document Reproduction Service No. ED 501717

Sunstein CR (2005) Moral heuristics. Behav Brain Sci 28(4):531-542. https:// doi.org/10.1017/S0140525X05000099. discussion 542-573

Sütterlin S, Herbert C, Schmitt M, Kübler A, Vögele C (2011) Overcoming selfishness: reciprocity, inhibition, and cardiac-autonomic control in the ultimatum game. Front Psychol 2:173. https://doi.org/10.3389/fpsyg.2011.00173

Telle N-T, Pfister H-R (2016) Positive empathy and prosocial behavior: a neglected link. Emotion Rev 8(2):154-163

Tibi-Elhanany Y, Shamay-Tsoory S (2011) Social cognition in social anxiety: first evidence for increased empathic abilities. Israel J Psychiatry Relat Sci 48(2):98

Tice DM, Bratslavsky E, Baumeister RF (2001) Emotional distress regulation takes precedence over impulse control: If you feel bad, do it! J Personal Soc Psychol 80(1):53

Turiel E (2015) Morality and prosocial judgments. The Oxford handbook of prosocial behavior, 137-152

Van Bavel JJ, Baicker K, Boggio PS, Capraro V, Cichocka A, Cikara M, Willer R (2020) Using social and behavioural science to support COVID-19 pandemic response. Nature human behav 4(5):460-471. https://doi.org/10.1038/s41562020-0884-z

Van Bavel JJ, Cichocka A, Capraro V, Sjåstad H, Nezlek JB, Alfano M, Ross RM (2021) National identity predicts public health support during a global pandemic: results from 67 nations. Nat Commun, Accepted

van de Groep S, Zanolie K, Green KH, Sweijen SW, Crone EA (2020) A daily diary study on adolescents' mood, empathy, and prosocial behavior during the COVID-19 pandemic. PLoS ONE 15(10):e0240349

Wang Y, Shi L, Que J, Lu Q, Liu L, Lu Z, Shi J (2021) The impact of quarantine on mental health status among general population in China during the COVID19 pandemic. Mol Psychiatry. https://doi.org/10.1038/s41380-021-01019-y
Witte K, Allen M (2000) A meta-analysis of fear appeals: implications for effective public health campaigns. Health Educ Behav 27(5):591-615

Yang S, Cho S-I (2017) Middle East respiratory syndrome risk perception among students at a university in South Korea, 2015. Am J Infect Control 45(6): e53-e60

Zainal NH, Newman MG (2018) Worry amplifies theory-of-mind reasoning for negatively valenced social stimuli in generalized anxiety disorder. J Affect Disord 227:824-833

Zaki J, Ochsner KN, Ochsner K (2012) The neuroscience of empathy: progress, pitfalls and promise. Nat Neurosci 15:675-680. https://doi.org/10.1038/ nn. 3085

Zettler I, Schild C, Lilleholt L, Kroencke L, Utesch T, Moshagen M, Geukes K (2020) The role of personality in COVID-19-related perceptions, evaluations, and behaviors: findings across five samples, nine traits, and 17 criteria. Soc Psychol Personal Sci https://doi.org/10.1177/19485506211001680

\section{Author contributions}

HS-G, MB, DF, MC, JM, JL, JM, JGP, HE and AI developed the study concept and the study design. HSG, MC, JM, JL, JM, SC, JP and IM performed testing and data collection. HS-G, AL, PS, JDL, SB performed the data analysis and interpretation under the supervision of AI. HS-G and AI drafted the manuscript. HS-G, MB, AL, DF, MC, JM, JL, JM JM-C, DMA, JCC, HS-G, DP, MLG-G, MH, AMG, JGP, SB, HE and AI provided critical revisions. All authors approved the final version of the manuscript for submission. All authors ensured that questions related to the accuracy or integrity of any part of the work are appropriately investigated and resolved.

\section{Competing interests}

The authors declare no competing interests.

\section{Ethical approval}

The study was approved by the Javeriana University ethical committee (code 2020/133) and conducted in accordance with the Declaration of Helsinki.

\section{Informed consent}

Subjects gave informed consent by pressing an "I agree" button beneath an explanatory letter informing potential respondents of the anonymity of their responses. All participants provided informed consent before the experimental procedures. After that, participants undertook the experiment individually.

\section{Additional information}

Supplementary information The online version contains supplementary material available at https://doi.org/10.1057/s41599-021-01022-2.

Correspondence and requests for materials should be addressed to Agustín. Ibanez.

Reprints and permission information is available at http://www.nature.com/reprints

Publisher's note Springer Nature remains neutral with regard to jurisdictional claims in published maps and institutional affiliations.

Open Access This article is licensed under a Creative Commons Attribution 4.0 International License, which permits use, sharing, adaptation, distribution and reproduction in any medium or format, as long as you give appropriate credit to the original author(s) and the source, provide a link to the Creative Commons license, and indicate if changes were made. The images or other third party material in this article are included in the article's Creative Commons license, unless indicated otherwise in a credit line to the material. If material is not included in the article's Creative Commons license and your intended use is not permitted by statutory regulation or exceeds the permitted use, you will need to obtain permission directly from the copyright holder. To view a copy of this license, visit http://creativecommons.org/ licenses/by/4.0/

(C) The Author(s) 2022 